\title{
Joseph Boussinesq (1842-1929) - un hydraulicien mathématicien
}

\author{
par Willi H. Hager et Félix Raemy \\ Laboratoire de recherches hydrauliques, hydrologiques et glaciologiques, \\ Ecole polytechnique fédérale de Zurich
}

\begin{abstract}
Boussinesq est considéré comme l'un des grands spécialistes français de la mécanique et de la physique mathématique. Ses travaux en hydrodynamique constituent également une partie importante de son ouvre. En 1897, il y a 100 ans, il apporta une contribution déterminante à la connaissance de la turbulence et de la couche limite par la publication de son ouvrage intitulé "Théorie de l'écoulement tourbillonnant et tumultueux des liquides". Dans ce qui suit, les étapes marquantes de sa vie et de son auvre sont retracées. Un hommage est ainsi rendu à ce savant relativement peu connu comme hydromécanicien, dont la vie se déroula dans le calme et la solitude.
\end{abstract}

\section{I — LA VOIE DE L'UNIVERSITÉ}

Joseph Valentin Boussinesq naquit le 13 mars 1842 à Saint-André-de-Sangonis (Dép. de l'Hérault). Son père, Jacques, était cultivateur. Sa mère, Anne-Marie Cavalier, fille d'un industriel, mourut en 1857. Joseph avait un frère plus jeune. A l'âge de 7 ans, il entra à l'école primaire du village où l'instituteur, à l'occasion de longues promenades, le rendit attentif à de nombreux phénomènes de la nature. Son oncle, un prêtre, lui enseigna le latin et le grec. Cependant, la jeunesse de Boussinesq reste peu connue.

Dès 16 ans, il fréquenta le lycée de Montpellier et devint maître-assistant. Bien qu'il suivît parallèlement des cours de mathématiques et de mécanique, il était essentiellement autodidacte. Jour et nuit, il se consacra à des travaux scientifiques, aussi bien à lâge de 19 ans où il obtint sa licence en mathématique que plus tard dans sa vie. Passionné par la mécanique et par la physique mathématique, il s'intéressa également à des questions religieuses et philosophiques. Les travaux de Fourier, de Cauchy, et plus généralement ceux des physiciens du début du XIXe siècle, semblent l'avoir complètement accaparé. Il devint lui-même une des dernières personnalités des sciences classiques de ce siècle-là, et acquit avant tout une renommée exceptionnelle en mécanique.

A 20 ans, il enseigna au collège d'Agde. Cette activité lui laissa beaucoup de temps pour étudier la littérature, entre autres les ouvrages de Lamé, ce qui le conduisit à publier son premier travail dans les "Comptes Rendus" de l'Académie. De manière élégante, Boussinesq y résolut un problème décrivant l'impact d'un jet d'eau sur une plaque, la capillarité étant prise en considération.

Ayant quitté Agde, Boussinesq exerça son activité au collège du Vigan puis à Gap (Dép. du Dauphiné). Dans cette ville, il épousa en 1869 la fille d'un officier de Mende (Lozère), Jeanne Giscard de la Roque (16 novembre 1819-14 août 1894). A cette époque, il travailla à la théorie de la lumière et aux lois de la dispersion. En 1867, Boussinesq présenta sa thèse de doctorat sur la propagation de la chaleur en milieu hétérogène à l'Académie des Sciences de Paris. Cette étude apporta une extension importante de la théorie de Lamé. En même temps, il envoya à l'Académie une

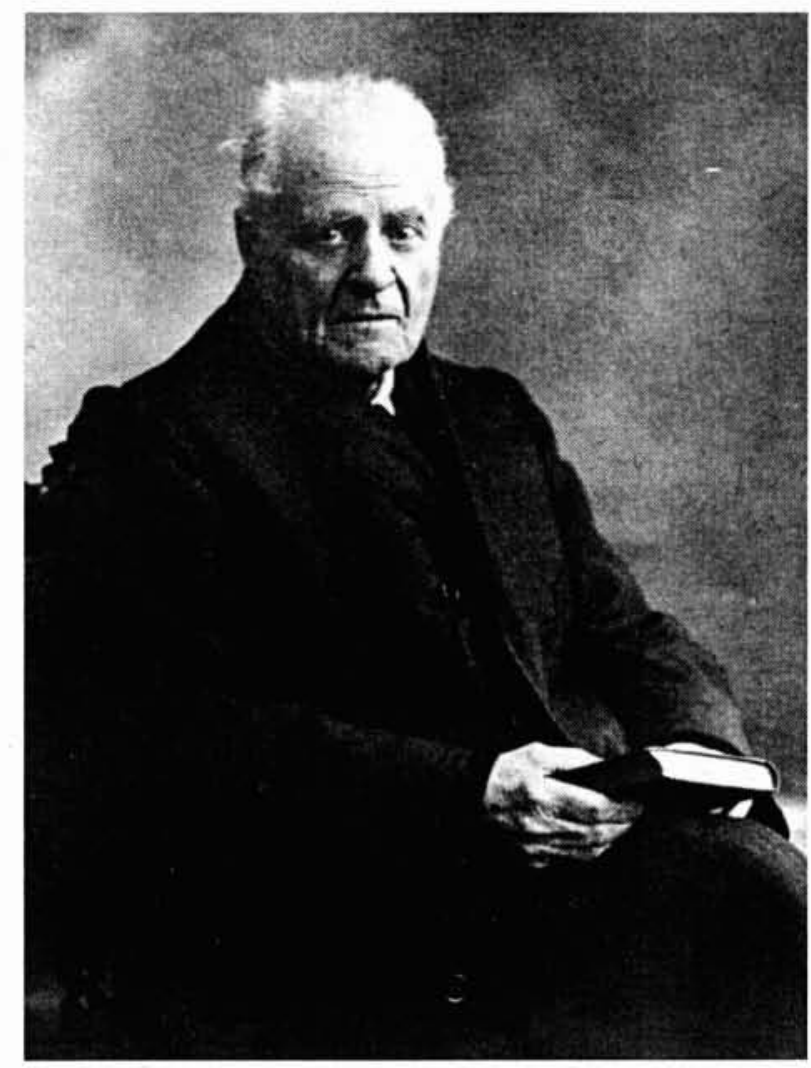

publication sur les petits mouvements des corps élastiques soumis à une sollicitation exercée dans les trois directions principales. Plusieurs années auparavant, De Saint-Venant (1797-1886) s'était également penché sur cette question et, partant d'hypothèses simplificatrices, avait trouvé la répartition elliptique des tensions. Boussinesq aboutit à une solution plus générale qui provoqua l'admiration du célèbre doyen de la mécanique. Dès ce moment, De Saint-Venant offrit un soutien inconditionnel au jeune chercheur de Gap. 
Selon le mathématicien Picard (1936), Boussinesq a apporté une contribution éminente dans tous les domaines de la physique mathématique, hormis celui de l'électromagnétisme. Son action a touché à la mécanique des sols, à l'optique, à l'étude de la chaleur, à la théorie de l'élasticité, à celle de la lumière, en mathématiques à la théorie des équations différentielles et même à la philosophie. Son style d'écriture était loin d'être simple et souvent des annotations pouvaient être plus longues que le texte lui-même. Boussinesq occasionnait des cauchemars aux compositeurs-typographes, ses corrections, renvoyées après lecture, atteignan parfois une longueur triple de celle du texte original. Conformément à sa nature, il était plein d'idées. Ses textes, très détaillés et faits de phrases compliquées, restent toutefois difficiles à saisir. L'introduction à la théorie de l'écoulement des liquides par les orifices en mince paroi (Boussinesq 1892) est un exemple significatif: "Lorsqu'un vase plein de liquide est percé, à travers une partie plane et mince de sa paroi, d'un orifice $\sigma$, de dimensions très petites par rapport à ses distances tant aux bords de cette partie plane qu'au reste de la surface limitant dans le vase la masse liquide, la vitesse $\mathrm{V}$ de celle-ci, à chaque instant et en chaque point intérieur, dépend assez simplement des volumes dq que débitent par unité de temps, à l'époque considérée, les divers éléments superficiels d $\sigma$ de l'orifice, et des distances $\mathrm{r}$ de ceux-ci do au point intérieur dont il s'agit ; car elle est la résultante de vitesses ou d'appels élémentaires, dirigés vers les éléments respectifs do et égaux à $\mathrm{dq} /\left(2 \pi \mathrm{r}^{2}\right)^{\prime}$. Les ouvrages cités en annexe ont souvent des titres qui caractérisent son style.

Pendant un séjour dans les Alpes françaises vers 1868 , Boussinesq commença à s'intéresser à l'hydrodynamique. L'échange de correspondance avec De Saint-Venant l'y avait conduit en toute logique. Ce savant était quasiment la seule personne avec laquelle Boussinesq discutait de questions techniques. Il montra alors un intérêt prononcé pour un poste à l'Université. Mais il devait au préalable acquérir une licence en physique. Ainsi, Boussinesq fut mis au bénéfice d'un congé par son collège en 1872 et une année plus tard, soit en 1873, De Saint-Venant obtint sa nomination comme professeur à la Faculté des Sciences de Lille.

\section{II — ÉVOLUTION DE L'HYDRODYNAMIQUE}

Alors que selon Leonhard Euler (1707-1783) les équations du mouvement des fluides pouvaient être décrites à l'aide de la théorie des potentiels, Louis Marie Henri Navier (17851836) tint de plus compte en 1822 de l'influence de la viscosité. Gotthilf Heinrich Ludwig Hagen (1797-1884) et Jean Louis Poiseuille (1799-1869) furent à même de résoudre le système d'équations de l'écoulement laminaire en conduite vers 1840 . Dès 1867, Boussinesq examina le problème de l'écoulement turbulent en conduite. Il avait connaissance des expériences d'Henri Emile Bazin (1865) et reconnut l'origine de la formation des tourbillons dans la viscosité. Contrairement à Navier, respectivement à George Gabriel Stokes (1819-1903), Boussinesq déduisit pourtant que l'action de la viscosité ne dépend pas uniquement du fluide, mais aussi de la position dans l'écoulement et du taux de turbulence (agitation tourbillonnaire). Il réussit à décrire mathématiquement les expériences de Bazin pour des cas simples. Dans sa publication parue en 1880, respectivement dans la "Théorie de l'écoulement tourbillonnant et tumultueux des liquides" (1897) et en particulier dans son "Essai sur la théorie des eaux courantes" (1877), le calcul montra une concordance étonnante avec le résultat expérimental. Cette démarche nécessitait une grande sûreté dans le choix des hypothèses, dans la simplification de systèmes complexes d'équations et dans l'intuition d'utiliser une approche analytique adéquate du phénomène. Ce sont justement ces propriétés particulières qui caractérisent les travaux de Boussinesq. Son mérite essentiel dans la théorie de la turbulence est d'avoir reconnu le problème de base, alors que de nombreux prédécesseurs n'avaient pas trouvé un point de départ. Ludwig Prandtl (1875-1953) fut toutefois le premier à fournir une contribution à l'étude de l'écoulement turbulent. A la parution, en 1904, de son travail déterminant consacré à la couche limite hydrodynamique, Prandtl attribua à Boussinesq l'introduction du coefficient de viscosité turbulente A dans la description de la tension de cisaillement apparente. De ce fait, le nom de Boussinesq reste indissolublement lié à la théorie de l'écoulement turbulent.

Le nom de Boussinesq est aussi lié aux ondes de translation, et en particulier à l'onde solitaire. Celle-ci peut être simplement engendrée par l'immersion d'un corps dans le fluide, par des manœuvres brusques d'arrêt d'un bateau ou encore par d'autres mécanismes d'excitation en canal découvert à section rectangulaire. L'onde solitaire est extrêmement riche en énergie et la hauteur de sa crête ne diminue que lentement sous l'effet de la viscosité. Elle a été décrite pour la première fois en 1834 par un Anglais, John Scott-Russell (1808-1882), qui observa l'arrêt brusque de bateaux. Différents chercheurs anglais renommés tentèrent d'expliquer mathématiquement le phénomène, afin de donner une assise théorique aux mesures de Scott-Russell. Ce fut Boussinesq qui le premier atteignit ce but, après qu'il eut introduit l'effet de la courbure des lignes de courant dans l'équation du mouvement. Il obtint une parfaite concordance entre ses calculs et les expériences anglaises. Cinq ans plus tard, Lord Rayleigh (1876) aboutit au même résultat par un autre cheminement.

Boussinesq s'est également occupé de différents types d'ondes, par exemple des ondes en eau peu profonde, des ondes oscillatoires ou des ondes de submersion. Seule la combinaison d'un outil mathématique de premier ordre avec une perception judicieuse du phénomène physique a permis de réduire les systèmes complexes d'équations à des approximations utilisables, auxquelles les méthodes mathématiques ont donné une solution. Dans ce métier, Boussinesq était un maître et l'on s'étonne encore aujourd'hui comme ingénieur, de voir à quel point la solution trouvée était généralement simple, bien que des pages de calculs compliqués aient été nécessaires à son obtention. La comparaison de la théorie avec l'expérimentation était particulièrement appréciée de Boussinesq, qui fit fréquemment appel au grand expérimentateur de l'époque : Henri Bazin. Les études importantes que Boussinesq entreprit au sujet de l'écoulement sur les déversoirs en sont un bel exemple. Les deux hommes étaient liés par une profonde amitié, comme Eydoux (1923) le rappela dans la nécrologie de Bazin.

Ce n'est qu'en 1902 que Boussinesq prit en considération l'influence de la température dans les problèmes de l'hydrodynamique. Il se référa aux physiciens français Fourier et Poisson dans la recherche des équations de base mais étudia essentiellement les méthodes de résolution des systèmes complexes d'équations. Il porta son intérêt sur la question de la convection de chaleur dans le système "corps solide-écoulement gazeux", par exemple sur le comportement des fluides au refroidissement. Il fut amené ainsi à la définition de la couche limite, considérée comme une zone de transition très mince entre le corps solide et l'écoulement du fluide, respectivement entre deux écoulements de fluides. Il mentionna l'effet important de la viscosité, entre autres sur la chute de gouttes d'eau dans l'air.

Parallèlement à l'hydrodynamique, Boussinesq se consacra aussi à la théorie de l'élasticité. Celle-ci fut établie en 1821 par Navier et suppose l'isotropie du corps solide avant sa déformation. La théorie fut ensuite développée par des spécialistes français de la mécanique, tels que Cauchy, Poisson, De Lamé et De Saint-Venant. Boussinesq trouva pourtant la réponse à la question de savoir comment la pression se répartit dans le milieu situé sous un corps reposant sur une 
surface plane. La solution de ce problème fut obtenue à partir d'une hypothèse simple concernant le potentiel. Boussinesq participa également au développement de la théorie du corps élastique. Là aussi, il fit des suppositions judicieuses sur la variation des tensions dans les directions transversale et longitudinale. De tels problèmes, qui ne pouvaient être résolus exactement par le calcul, convenaient naturellement au physicien-mécanicien Boussinesq. Par exemple, il réussit à formuler les équations généralisées et interdépendantes exprimant la tension et la torsion, après avoir subdivisé une plaque en prismes élémentaires. En 1953, Timoshenko rendit un vibrant hommage à Boussinesq pour ses mérites. Il présenta l'extension de la théorie de De Saint-Venant et rappela qu'avec Maurice Lévy (1838-1910) et Alfred Aimé Flamant (1839-1914), Boussinesq avait été l'élève de De SaintVenant.

\section{III a CONTRIBUTION À L'ÉTUDE DE LA TURBULENCE}

Les premiers résultats concernant les écoulements en conduite furent publiés par Poiseuille (1846), qui montra avec justesse, tant par l'expérience que par la théorie, les caractéristiques du régime laminaire. Il fut le premier à formuler la loi de vitesse correspondante

$$
\mathrm{V}=2 \mathrm{gJD}^{2} /(64 v)
$$

où $\mathrm{V}$ représente la vitesse moyenne, $\mathrm{g}$ l'accélération de la pesanteur, J la pente de frottement, D le diamètre de la conduite et $v$ la viscosité cinématique. Boussinesq (1897) rendit toutefois attentif à la grandeur énorme des vitesses avec lesquelles l'écoulement aurait dû traverser une conduite de grandes dimensions, si l'équation (1) était encore valable dans ce cas. En admettant par exemple une pente de frottement de $0,1 \%$, un diamètre de $1 \mathrm{~m}$ et une viscosité cinématique $\mathrm{v}=10^{-6} \mathrm{~m}^{2} \mathrm{~s}^{-1}$ pour de l'eau à $20^{\circ} \mathrm{C}$, la vitesse $\mathrm{V}$ serait égale à $20 \times 10^{-3} \times 1 /\left(64 \times 10^{-6}\right)=312 \mathrm{~ms}^{-1}$ soit pratiquement la vitesse du son dans l'air. Aux grandes vitesses, les résistances à l'écoulement sont par conséquent nettement supérieures à celles agissant en régime laminaire.

Darcy et plus tard Reynolds (1883) avaient étudié la transition de l'écoulement laminaire à l'écoulement turbulent. Celle-ci dépend fortement du nombre de Reynolds $\mathrm{R}=\mathrm{VD} / \mathrm{v}$. Alors que Reynolds parlait encore de "sinuous motion", Boussinesq appela le phénomène "écoulement tourbillonnant et tumultueux" tandis que Boulanger (1909), qui a présenté un résumé des travaux hydrauliques de Boussinesq, utilisa l'expression "régime turbulent." Selon Boulanger, les écoulements turbulents reflètent les phénomènes les plus importants de l' hydraulique. Si cette turbulence ne se développait pas dans les cours d'eau, leur surface serait lisse comme de la glace. Sans les investigations de Boussinesq, la turbulence ne serait restée, selon De Saint-Venant, qu'une désespérante énigme. Euler, déjà, avait reconnu l'influence de la paroi et de la viscosité du fluide comme causes de l'apparition de l'écoulement caractéristique appelé aujourd'hui "turbulence". Ce fut pourtant Boussinesq qui parvint à une première quantification de la turbulence.

Afin de décrire le phénomène, il distingua entre les valeurs moyennes locales temporelles de grandeurs telles que la pression et la vitesse, et les valeurs de leurs fluctuations (agitation locale). Ces dernières sont généralement faibles mais montrent d'importantes variations dans le temps. La somme des fluctuations, prise sur une séquence donnée, est nulle par définition. En 1896, Boussinesq établit, sans en avoir connu au préalable l'existence, les équations du mouvement fluctuant de Reynolds (1895) puis tenta de quantifier le frottement interne. Dans un écoulement turbulent, la grandeur désignée par $\varepsilon$ est uniquement dépendante de l'agitation tourbillonnaire. Boussinesq considéra deux cas particuliers, à savoir la conduite circulaire et le canal rectangulaire large. Il s'agit de profils dans lesquels la vitesse moyenne axiale $u$ est beaucoup plus grande que les vitesses transversales $\mathrm{v}$ et $\mathrm{w}$. Les accélérations de $\mathrm{v}$ et $\mathrm{w}$ en direction longitudinale sont négligeables.

En 1877 déjà, Boussinesq rechercha les causes de la production de turbulence. En observant la surface libre d'une rivière, il remarqua la présence répétée de "balles de fluide" se dirigeant de la rive vers l'axe du cours d'eau en formant de petits tourbillons. De plus il constata, particulièrement au voisinage de l'axe, des mouvements tourbillonnaires venus du fond et y retournant. L'origine de la turbulence est donc à chercher à la paroi délimitant le fluide. Selon l'expérience, la grandeur de cette turbulence varie fortement avec la vitesse locale, avec le rayon hydraulique comme indice de la délimitation du fluide par la paroi et avec la rugosité de paroi elle-même.

Sur la base de cette analyse, Boussinesq pose le facteur $\varepsilon$ égal au produit de la vitesse à la paroi $u_{0}$ par le rayon hydraulique $R_{h}$, par la masse spécifique $\rho$ du fluide, par l'accélération de la pesanteur $g$ et finalement par un coefficient A* caractérisant la rugosité de la paroi. On a ainsi pour le profil rectangulaire

$$
\varepsilon=\rho g \mathrm{~A}^{*} \mathrm{hu}_{\mathrm{o}}
$$

avec $\mathrm{h}$, la profondeur d'eau comme rayon hydraulique d'un canal rectangulaire large. Pour le profil circulaire, il résulte

$$
\varepsilon=\rho g A * \frac{D}{4} u_{o}(R / r) .
$$

Avec la coordonnée radiale $\mathrm{r}$ rapportée au rayon $\mathrm{R}$, l'accroissement de $\varepsilon$ entre la paroi et le centre de la conduite est mis en évidence.

En désignant par $\mathrm{J}$ la pente de la ligne piézométrique, la composante axiale de l'équation de Navier-Stokes devient, pour l'écoulement uniforme dans un canal rectangulaire large

$$
\rho g J+\varepsilon \frac{\partial^{2} u}{\partial z^{2}}=0
$$

où $\mathrm{z}$ est la coordonnée mesurée de la surface libre perpendiculairement au radier du canal. En remplaçant $\varepsilon$ par sa valeur donnée dans l'équation (2), on obtient

$$
\mathrm{J}+\mathrm{A}^{*} \mathrm{u}_{\mathrm{o}} \mathrm{h} \frac{\partial^{2} \mathrm{u}}{\partial \mathrm{z}^{2}}=0
$$

Comme le frottement est nul à la surface, on a après la première intégration

$$
\mathrm{Jz}+\mathrm{A} * \mathrm{u}_{\mathrm{o}} \mathrm{h} \frac{\partial \mathrm{u}}{\partial \mathrm{z}}=0
$$

Sur le fond où $\mathrm{z}=\mathrm{h}$, le frottement doit être égal au frottement de paroi. Il suit que $\mathrm{Jh}=\left(\mathrm{KA}^{*} \mathrm{u}_{\mathrm{o}}\right)^{2}$, où $\mathrm{K}\left[\mathrm{m}^{1 / 2} \mathrm{~s}^{-1}\right]$ est une mesure pour la rugosité de paroi. En éliminant J dans (6), on trouve

$$
\mathrm{K}^{2} \mathrm{~A} * \mathrm{u}_{\mathrm{o}} \frac{\mathrm{z}}{\mathrm{h}^{2}}+\frac{\partial \mathrm{u}}{\partial \mathrm{z}}=0
$$

Après intégration avec la condition aux limites $u(z=h)=u_{o}$, la solution est (Boussinesq 1897)

$$
\left(\frac{\mathrm{u}}{\mathrm{u}_{\mathrm{o}}}-1\right)=\frac{1}{2} \mathrm{~K}^{2} \mathrm{~A} *\left(1-\frac{\mathrm{z}^{2}}{\mathrm{~h}^{2}}\right)
$$


Cette relation peut être présentée sous la forme actuelle

$$
\frac{\mathrm{u}_{\mathrm{M}}-\mathrm{u}}{(\mathrm{hJ})^{1 / 2}}=\frac{\mathrm{K}}{2}\left(\frac{\mathrm{z}}{\mathrm{h}}\right)^{2}
$$

avec $\mathrm{u}_{\mathrm{M}}$ la vitesse maximale. Le coefficient $\mathrm{K}$ est de $44,55 \mathrm{~m}^{1 / 2} \mathrm{~s}^{-1}$.

Effectuant des calculs analogues pour le profil circulaire, Boussinesq (1897) arrive au résultat suivant

$$
\frac{\mathrm{u}_{\mathrm{M}}-\mathrm{u}}{(\mathrm{JD})^{1 / 2}}=\frac{\mathrm{K}}{2}\left(\frac{\mathrm{r}}{\mathrm{R}}\right)^{3}
$$

avec $\mathrm{K}=48,6 \mathrm{~m}^{1 / 2} \mathrm{~s}^{-1}$. Les relations établies pour les profils rectangulaire et circulaire coïncident qualitativement de manière parfaite avec les mesures de Bazin (1865). Les écarts sur les coefficients $\mathrm{K}$ sont de l'ordre de $10 \%$. Lors d'essais ultérieurs effectués dans des conduites circulaires de diamètre $0,8 \mathrm{~m}$, Bazin (1902) confirma les résultats de Boussinesq, en particulier la dépendance cubique avec la distance au centre. Certains écarts furent uniquement constatés au voisinage de l'axe de la conduite, et Boussinesq améliora sa théorie afin de supprimer la singularité contenue dans l'équation (3).

La théorie de Boussinesq fut révolutionnaire pour son époque. On avait ainsi acquis des méthodes permettant de relier la théorie classique aux expériences. En particulier, un regain de confiance se manifesta pour les équations générales du mouvement de Navier-Stokes. Un rapport important allait se créer entre la démarche de la pensée et la vérification découlant de la mesure expérimentale. La proposition de Boussinesq pour la répartition de la grandeur $\varepsilon$ fut confirmée par Hahn et autres (1904) dans la zone centrale de l'écoulement, alors que sur la base des mesures de Bazin (1902), ils constatèrent une forte diminution en direction de la paroi.

Dans l'optique actuelle, Bazin et Boussinesq ont commis une erreur d'appréciation en admettant une vitesse $u_{\mathrm{o}}$ à la paroi. La condition d'adhérence à la paroi de l'hydrodynamique, démontrée en premier par Hele-Shaw vers 1900, n'était ici pas remplie. Bien entendu, les méthodes expérimentales d'il y a 100 ans étaient trop imprécises pour explorer la couche limite d'un écoulement et y déceler la présence de la sous-couche laminaire. Ce détail important n'échappa sans doute pas à Boussinesq mais, comme la concordance avec les observations avait été jugée généralement suffisante et que la technique de mesure dans la couche limite n'était pas développée, il se contenta de cette approche.

En 1904, Prandtl permit de faire un grand progrès dans la question de la couche limite. Faisant intervenir le nombre de Reynolds et normalisant l'épaisseur $\delta$ de la couche limite par une longueur typique $\mathrm{L}$, il obtint la relation $\delta / L=R^{-1 / 2}$. L'apport déterminant dans la théorie de la couche limite est dû à Prandtl (1933) et à Von Karman (1930). Par analogie avec la loi de frottement de l'écoulement laminaire où la tension de cisaillement est

$$
\tau=\mu \frac{\partial u}{\partial z}
$$

Boussinesq introduit la tension de cisaillement apparente

$$
\tau^{\prime}=\mathrm{A} \frac{\partial \overline{\mathrm{u}}}{\partial \mathrm{z}}=\rho \varepsilon \frac{\partial \mathrm{u}}{\partial \mathrm{z}}
$$

Ici, A est un coefficient de viscosité turbulente et $\overline{\mathrm{u}}$ la moyenne temporelle de la vitesse longitudinale. La viscosité cinématique $v=\mu / \rho$ de l'écoulement laminaire correspond ainsi à la viscosité turbulente $\varepsilon=\mathrm{A} / \rho$ de l'écoulement turbulent. L'hypothèse de Boussinesq présente cependant le désavantage que $\varepsilon$ n'est pas une constante du fluide comme $v$, mais dépend de la valeur du gradient de la vitesse. Un pas décisif fut fait en 1925 par Prandtl à l'aide d'une considéra- tion sur la quantité de mouvement, dans laquelle il introduisit la longueur de mélange $\lambda$. Comme résultat, il obtint la relation $\varepsilon=\lambda^{2}|\mathrm{~d} \overline{\mathrm{u}} / \mathrm{dz}|$, où $\lambda$ est indépendant de la grandeur de la vitesse. La longueur $\lambda$ put alors être exprimée par le produit $\kappa y$, avec $\mathrm{y}$ la distance à la paroi et $\kappa$ une constante adimensionnelle. Finalement, Prandtl aboutit à la répartition logarithmique des vitesses dans la section d'écoulement.

Ce fut toutefois Boussinesq qui posa les bases de cette théorie, aujourd'hui classique, de la couche limite. En 1897, soit il y a juste 100 ans, il avait publié en effet son important ouvrage sur la "Théorie de l'écoulement tourbillonnant et tumultueux des liquides".

\section{IV घ VIE ET GEUVRE À PARIS}

Au printemps 1886, Boussinesq fut nommé professeur de mécanique à l'Académie des Sciences de Paris. Malheureusement, son mentor De Saint-Venant mourut dix jours plus tôt, de sorte qu'une collaboration directe à Paris ne put avoir lieu. La nécrologie de De Saint-Venant, rédigée par Boussinesq et Flamant, fit état d'une affection personnelle pour le grand maître.

Touché et stimulé par la disparition de ce dernier, Boussinesq se mit à examiner le problème de la transmission des chocs dus à un impact, comme il est posé par des projectiles atteignant une cible. Il était toutefois nécessaire d'étudier au préalable la propagation des ondes dans les corps solides. Boussinesq reprit d'autre part ses investigations concernant le problème de la pression exercée par les terres, problème que Charles Augustin de Coulomb (1736-1806) avait abordé au XVIIIe siècle. Plus tard, Rankine, De Saint-Venant et Lévy développèrent la théorie ne faisant plus intervenir une surface de rupture plane. Boussinesq remplaça le coefficient d'élasticité admis jusqu'alors constant par un autre coefficient variant proportionnellement à la pression moyenne. En 1896, Boussinesq devint professeur en physique mathématique et en théorie des probabilités.

Une année après la mort de Jeanne, sa première femme, Boussinesq épousa le 29 août 1895 Claire Onffroy de Vérez, fille du baron de Vérez. Celle-ci devait décéder le $24 / 11 / 1905$. Finalement il épousa, vers 1906, Jeanne Le Bouteiller, de laquelle il divorça au début de 1909. Ces unions restèrent sans enfants et aucune information sur la vie familiale de Boussinesq n'est disponible.

La plupart de ses travaux scientifiques parurent dans des revues spécialisées françaises, comme le "Journal de Mathématique", les "Comptes rendus de l'Académie des Sciences" ou dans les "Annales des Ponts et Chaussées", ainsi que dans les "Mémoires de l'Académie". Poggendorf en a établi une bibliographie détaillée. Boussinesq enseigna à la Sorbonne pendant plus de trente ans jusqu'à sa retraite en 1918. L'ouvrage en trois parties, qui parut entre 1901 et 1922, traitait des "Leçons sur la Mécanique et la Physique Mathématique". Boussinesq fut honoré en 1871 par l'octroi du Prix Poncelet. En 1886, il devint membre de l'Académie des Sciences et accéda, en 1901, au grade d'Officier de la Légion d'Honneur. Sa vie fut ainsi consacrée à la recherche, à l'étude de littérature, à la méditation sur des questions religieuses et naturellement à la rédaction de publications. Sur sa table de travail, on trouvait les livres de Platon et d'Aristote ou encore ceux de Thomas d'Aquin. Chaque jour à 15 heures, Boussinesq se rendait à la bibliothèque, s'asseyait toujours à la même place qu'il ne quittait qu'à la fermeture. A la fin de sa vie, il vécut très retiré. La tendance à la tristesse et à la solitude qu'il avait déjà en lui depuis son jeune âge s'accentua avec les maladies de la vieillesse. Lui qui avait sans cesse eu peur du monde, se montrait très prudent en appréciant son entourage. Mais le jugement d'ensemble qu'il portait paraissait être positif. Boussinesq, catholique pratiquant, mourut le 19 février 1929 à l'âge de presque quatre-vingt sept ans. 


\section{BIBLIOGRAPHIE CHOISIE}

[1] BoussinesQ, J. (1868). Mémoire sur l'influence des frottements dans les mouvements réguliers des fluides. Journal de Mathématiques Pures et Appliquées 13(2): 377-423.

[2] BoussinesQ, J. (1871). Théorie de l'intumescence liquide appelée onde solitaire ou de translation, se propageant dans un canal rectangulaire. Comptes Rendus Académie des Sciences Paris 72: 755-759; 73: 256-260; 73: 1210-1212.

[3] BoussinesQ, J. (1877). Essai sur la théorie des eaux courantes. Mémoires présentés par divers savants à l'Académie des Sciences Paris 23(1): 1-660; 24(2): 1-60.

[4] BoussinesQ, J. (1878). Complément à une étude intitulée "Essai sur la théorie des eaux courantes", et à un mémoire "Sur l'influence des frottements dans les mouvements réguliers des fluides". Journal de Mathématiques Pures et Appliquées 4 Ser.3: 335-376.

[5] BoussinesQ, J. (1892). Sur la théorie de l'écoulement des liquides par les orifices en mince paroi, circulaires ou rectangulaires allongés; calcul approché du débit et de sa répartition entre les divers éléments superficiels de l'orifice. Journal de Physique Théorique et Appliquée 1 Ser.3: 265-285.

[6] BoussinesQ, J. (1897). Théorie de l'écoulement tourbillonnant et tumultueux des liquides [dans les lits rectilignes à grande section]. Gauthier-Villars et Fils: Paris.

[7] BoussinesQ, J. (1908). Théorie approchée de l'écoulement de l'eau sur un déversoir [en mince paroi et sans contraction latérale]. Mémoires de l'Académie des Sciences 50: $1-134$.

\section{Théorie de la turbulence}

[8] Bazin, H. (1865). Recherches expérimentales sur l'écoulement de l'eau dans les canaux découverts. Mémoires presentés par divers savants étrangers à l'Académie des Sciences Paris 19: 1-494.

[9] BAZIN, H. (1902). Expériences nouvelles sur la distribution des vitesses dans les tuyaux. Mémoires présentés par divers savants étrangers à l'Académie des Sciences Paris $32(6)$.

[10] Boulanger, A. (1909). Hydraulique générale. 1 Principes et problèmes fondamentaux. Octave Doin et Fils: Paris.

[11] Hahn, H., Herglotz, G., Schwarzschild, K. (1904). Uber das Strömen des Wassers in Röhren und Kanälen. Zeitschrift für Mathematik und Physik 51: 411-426.

[12] LoRd Rayleigh (1876). On waves. Philosophical Magazine 1(Series 5): 257-279.

[13] Poiseuille, J.L. (1846). Recherches expérimentales sur le mouvement des liquides dans les tubes de très petits diamètres. Mémoires présentés par divers savants étrangers à l'Académie des Sciences Paris 9: 433-543.
[14] PrandtL, L. (1904). Über Flüssigkeitsbewegung bei sehr kleiner Reibung. Verhandlungen 3. Internationaler Mathematiker-Kongress Heidelberg: 484-491.

[15] PRANDTL, L. (1925). Bericht über Untersuchungen zur ausgebildeten Turbulenz. Zeitschrift für Angewandte Mathematik und Mechanik 5(2): 136-139.

[16] PRANDTL, L. (1933). Neuere Ergebnisse der Turbulenzforschung. Zeitschrift VdI 77(5): 105-114.

[17] Reynolds, O. (1883). An experimental investigation of the circumstances which determine whether the motion of water shall be direct or sinuous, and of the law of resistance in parallel channels. Philosophical Transactions A174: 935982.

[18] Reynolds, O. (1895). On the dynamical theory of incompressible viscous fluids and the determination of the criterion. Philosophical Transactions A186: 123-164.

[19] Von Karman, T. (1930). Mechanische Ähnlichkeit und Turbulenz. Nachrichten der Gesellschaft für Wissenschaft Göttingen, Math.-Physikalische Klasse: 58-76.

\section{BIOGRAPHIES}

[20] Anonymus (1929). Nécrologie: Joseph Boussinesq. Revue Générale de l'Electricité 25(12): 433-434.

[21] Berger, H. et Ballot, C.B. (1970) Boussinesq, Joseph Valentin. Dictionary of scientific biography. Charles Scribner's Sons. New York.

[22] BoussinesQ, J. et Flamant, A. (1886). Notice sur la vie et les travaux de M. De Saint-Venant. Annales des Ponts et Chaussées 56(4): 557-595.

[23] Charle, C. et Telkes, E. (1989). Les professeurs de la faculté des sciences de Paris. Histoire Biographique de l'Enseignement. Dictionnaire biographique 1901-1939. Edition du CNRS: Paris.

[24] Eydoux, Y. (1923). A la mémoire d'Henri Bazin. La Houille Blanche 22: 52-56.

[25] Mangin, L. (1929). Nécrologie: Joseph Boussinesq. Revue générale des sciences pures et appliquées 40(7): 193194. Parue également dans les Comptes Rendus de l'Académie des Sciences Paris 188(9): 589-591.

[26] Marcolongo, R. (1929). Commemorazione di Valentino Giuseppe Boussinesq. Rendiconto dell'Academia delle Scienze fisiche e matematiche di Napoli 68: 114-115.

[27] PiCard, E. (1936). La vie et l'oeuvre de Joseph Boussinesq. Discours et Notices. Gauthier-Villars: Paris.

[28] Poggendorf, J.C. (1959). Biographisch-Literarisches Handwörterbuch der exakten Wissenschaften. Bände II, IV, V, VI. Akademie Verlag: Berlin.

[29] Timoshenko, S.P. (1953). History of strength of materials. McGraw-Hill: New York. 